\title{
ACTIONS OF FINITE GROUPS ON HOMOTOPY 3-SPHERES
}

BY

M. E. FEIGHN

\begin{abstract}
It is conjectured that the action of a finite group of diffeomorphisms of the 3-sphere is equivariantly diffeomorphic to a linear action. This conjecture is verified if both of the following conditions hold: (i) Each isotropy subgroup is dihedral or cyclic. (ii) There is a point with cyclic isotropy subgroup of order not $1,2,3$ or 5 .
\end{abstract}

0. Introduction. The generalized Smith conjecture says that the effective action of a finite group $G$ of orientation preserving diffeomorphisms on a homotopy 3-sphere $\Sigma$ is essentially linear. If $\Sigma$ is diffeomorphic to $S^{3}$, then "essentially linear" is equivalent to "equivariantly diffeomorphic to a linear action."

Work by Thurston, Johannson, Jaco, Shalen, Bass, Meeks and Yau established this conjecture when $G$ is generated by a diffeomorphism with fixed points. This is the classical Smith conjecture. Davis and Morgan improved this in certain cases by proving that if every isotropy subgroup of $G$ is cyclic and if at least one of these subgroups has order greater than 5 , then the action is essentially linear.

The group $G$ is finite and the action is differentiable and effective. Thus, the isotropy subgroup of a point in $\Sigma$ acts on the tangent space at the point and so is a finite subgroup of $\mathrm{GL}_{3}^{+}(\mathbf{R})$. Therefore this group is cyclic, dihedral, tetrahedral, octahedral or icosahedral.

In this paper, the result of Davis and Morgan will be extended to include the case where one of the isotropy subgroups has order 4 . We also show that the actions is essentially linear if every isotropy subgroup is either dihedral or cyclic and one of the cyclic isotropy subgroups has order not equal to $1,2,3$ or 5 .

The results of this paper first appeared in the author's 1981 thesis at Columbia University. Subsequently, William Thurston announced a theorem that includes these results as a special case.

For another proof of the Davis-Morgan extension of the Smith conjecture, see Culler and Shalen [1].

I would like to express my deepest gratitude to my thesis advisor, John Morgan. I would also like to thank Michael Davis.

I. Preliminaries. We start with pertinent definitions from orbifold theory and recall some results of Davis and Morgan [2]. Other results of that paper are extended to include the dihedral case. For more on orbifolds, see Thurston [5].

Received by the editors March 9, 1982 and, in revised form, June 20, 1983.

1980 Mathematics Subject Classification. Primary 57S17.

(C) 1984 American Mathematical Society $0002-9947 / 84 \$ 1.00+\$ .25$ per page 
Let $D^{n}=\left\{x \in \mathbf{R}^{n}|| x \mid<1\right\}$. An $n$-orbifold is a space locally modelled on $D^{n}$ modulo finite groups. To be more precise, an $n$-orbifold chart on the topological space $X$ is a triple $(U, G, h)$ where $U$ is an open subset of $X, G$ is a finite subgroup of $O(n)$, and $h: U \rightarrow D^{n} / G$ is a homeomorphism.

A (locally smooth) $n$-orbifold is a paracompact, Hausdorff space $X$ together with a collection $\mathscr{U}$ of $n$-orbifold charts which satisfies the following three conditions:

(1) The collection $\{U \mid \exists(U, G, h) \in \mathcal{Q}$. $\}$ is a basis for the topology on $X$.

(2) If $\left(U_{1}, G_{1}, h_{1}\right) \in \mathcal{Q},\left(U_{2}, G_{2}, h_{2}\right) \in \mathcal{Q}$ and $U_{1} \subset U_{2}$, then there is an embed$\operatorname{ding} f: D^{n} \rightarrow D^{n}$ such that the following diagram commutes:

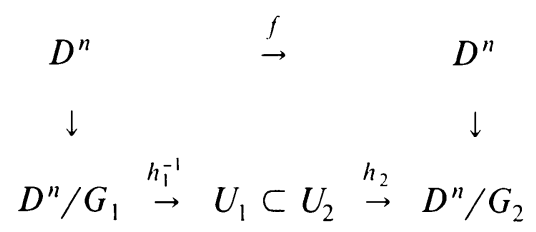

Note. Any other lift $f^{\prime}$ differs from $f$ by left multiplication in $G_{2}$. Further, a choice of lift $f$ determines a unique injective homomorphism $\varphi: G_{1} \rightarrow G_{2}$ such that $f$ is $\varphi$-equivariant.

(3) The collection $\mathcal{Q}$ is maximal with respect to condition (2).

For an orbifold with boundary, add charts of the form $D_{+}^{n} / G$ where $D_{+}^{n}=$ $\left\{\left(x_{1}, \ldots, x_{n}\right) \in D^{n} \mid x_{n} \geqslant 0\right\}$ and where $G \subset O(n)$ leaves the half-space $\left\{x_{n} \geqslant 0\right\}$ invariant.

An orbifold is smooth if the overlap maps in the definition are smooth.

The underlying space of an orbifold is the topological space without its orbifold structure.

If $(U, G, h)$ is an orbifold chart on the orbibold $X$ and $x \in U$, then the local group at $x$ is defined to be the conjugacy class in $O(n)$ of the stabilizer in $G$ of a point in the preimage of $h(x)$ under the projection $D^{n} \rightarrow D^{n} / G$. The exceptional set of $X$, denoted ex $(X)$, is the set of all points in $X$ with nontrivial local group.

An orientation for a chart $(U, G, h)$ is an orientation for $D^{n}$ in which $G$ acts as a group of orientation preserving maps. An orientation for an orbifold is a compatible system of orientations for the charts defining the orbifold structure.

A covering projection $p: Y \rightarrow X$ is a continuous map between the underlying spaces of $Y$ and $X$ with the following property. Each point in $X$ has a neighborhood which is the first coordinate of a chart $(U, G, h)$ on $X$ such that each component of $p^{-1}(U)$ is the first coordinate of a chart $(V, H, g)$ on $Y$ with $H \subset G$ making the diagram below commute:

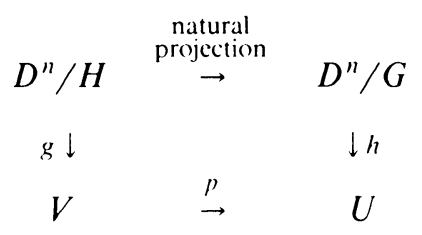


In short, covering projections are locally modelled on $\amalg D^{n} / G_{i} \rightarrow D^{n} / G$ where $G_{i} \subset G$.

An isomorphism $p: Y \rightarrow X$ is a map such that both $p$ and $p^{-1}$ are covering projections.

The usual covering space theory for topological spaces goes over to a covering space theory for orbifolds. For example, every orbifold has a universal cover and it is unique up to covering isomorphisms [5, Proposition 13.2.4]. Van Kampen's theorem also extends to orbifolds.

Let $\tilde{X} \rightarrow X$ be the universal covering projection for $X$. The group of covering isomorphisms of this cover is called the orbifold fundamental group of $X$ and is denoted by $\pi_{1}^{\text {orb }}(X)$.

A covering projection $Y \rightarrow X$ is regular (or normal) if $X$ is the quotient orbifold of the action of the covering transformations.

There is a one-to-one correspondence between subgroups of $\pi_{1}^{\text {orb }}(X)$ and connected orbifold covers of $X$. Regular covers correspond to normal subgroups.

An orbifold is good if it has a cover which is a manifold.

We now restrict our attention to dimension three. The finite subgroups of $\mathrm{SO}(3)$ and their quotients of $D^{3}$ are:

cyclic groups

dihedral groups
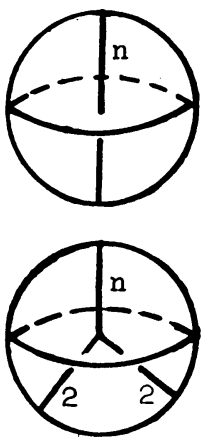

the tetrahedral group

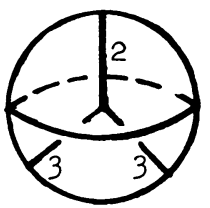

the octahedral group

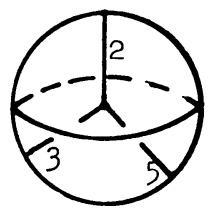

the icosahedral group

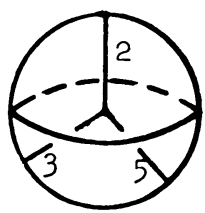


Thus, we see that the exceptional set of an orientable 3-orbifold is a trivalent graph. The graph may contain edges without vertices (knots). The condition at a vertex is that the sum of the reciprocals of the orders of the local groups of the incident edges is greater than one. If a local group is cyclic then we label the corresponding edge in the exceptional set by the order of this group.

The orbifold structure of an orientable 3-orbifold is determined up to isomorphism by its underlying space and its exceptional set (with labels). Given a 3-manifold together with an embedded, labelled trivalent graph satisfying the above vertex condition, there is an induced smooth orbifold structure. This structure is unique up to smooth isomorphism [2, section 2].

Let $X$ be a orientable 3-orbifold. Let $\Gamma=\pi_{1}(X-\operatorname{ex}(X))$. There is a surjection $i_{*}$ : $\Gamma \rightarrow \pi_{1}^{\text {orb }}(X)$. Label the edges and circles of $\operatorname{ex}(X)$ by $e_{1}, \ldots, e_{T}$. Let their labels be $\rho\left(e_{1}\right), \ldots, \rho\left(e_{T}\right)$, respectively. Let $\mu_{i} \in \Gamma$ be the class of the "meridian" about $e_{i}$. The kernel of $i_{*}$ is the subgroup of $\Gamma$ normally generated by $\left\{\left(\mu_{1}\right)^{\rho\left(e_{1}\right)}, \ldots,\left(\mu_{T}\right)^{\rho\left(e_{T}\right)}\right\}$ [2, section 3].

Seifert fibered orbifolds will bridge a gap between orbifolds and linear actions. A Seifert fibered orbifold $X^{3}$ is a 3-orbifold equipped with a map to a 2-orbifold, $X^{3} \rightarrow B^{2}$, which is locally modelled on $\left(S^{1} \times D^{2}\right) / G \rightarrow D^{2} / G$ where $G$ acts orthogonally on both factors and effectively on $D^{2}$. (If we allow $X$ to have boundary, then there the map is modelled on $\left(S^{1} \times D_{+}^{2}\right) / G \rightarrow D_{+}^{2} / G$.

An orientable 3-orbifold is dihedral if all the local groups are cyclic or dihedral. It follows immediately from the definition that an orientable, Seifert fibered orbifold is dihedral and that any edge or circle in the exceptional set with order larger than 2 is a fiber.

A dihedral 3-orbifold with finite fundamental group is good. (There are always cycles in the exceptional set with each edge having the same label. Thus, by taking covers corresponding to the fundamental group of an underlying space or cyclic branched covers over cycles, a succession of orbifold covers ending with a manifold can be found.)

If $X$ is Seifert fibered and orientable, then each fiber on the boundary of $X$ has a neighborhood on the boundary of the form $S^{1} \times I$ or $\left(S^{1} \times I\right) /(\mathbf{Z} / 2 \mathbf{Z})$ where $\mathbf{Z} / 2 \mathbf{Z}$ acts on a reflection on both factors and the fibration is induced by projection onto the second factor:
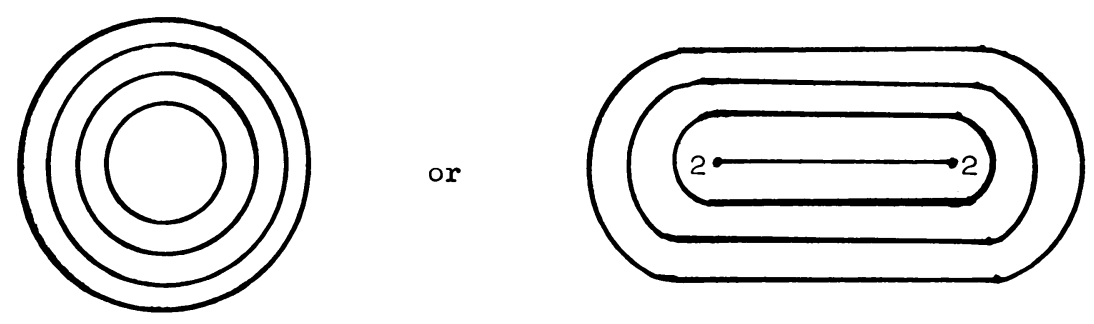

Therefore, if a boundary component is closed, then it is diffeomorphic to $S^{1} \times S^{1}$ or $\left(S^{1} \times S^{1}\right) /(\mathbf{Z} / 2 \mathbf{Z})$ where again the action is reflection in both factors and the 
fibration is induced as before. Denote $\left(S^{1} \times S^{1}\right) /(\mathbf{Z} / 2 \mathbf{Z})$ by $\bar{T}^{2}$, a 2 -sphere with 4 points labelled by 2 :

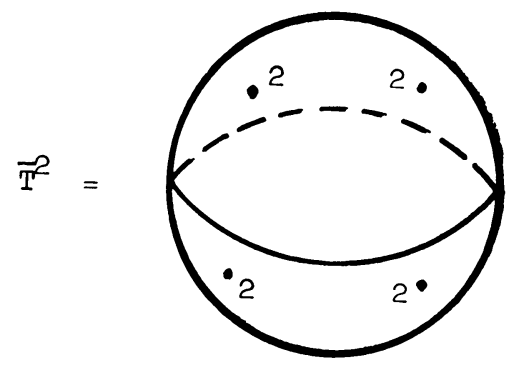

Our main tool is given by the following theorem of Davis and Morgan [2, Theorem 5.1].

TheOREM I.1. Assume $X$ is an orientable 3-orbifold with finite fundamental group. Suppose that $X$ is Seifert fibered; then there is a subgroup $G \subset \mathrm{SO}(4)$ so that $X$ and $S^{3} / G$ are diffeomorphic.

The following two propositions from Davis and Morgan [2] are useful in examining the behavior of Seifert fibrations under covering maps.

Theorem I.2 [2, Theorem 8.1]. Assume that $X$ is a prime, good, orientable 3-orbifold with nonempty boundary. Then $X$ is Seifert fibered iff $\pi_{1}^{\text {orb }}(X)$ contains a nontrivial, normal, cyclic subgroup.

Proposition I.3 [2, Proposition 8.2]. Let $X$ be as in Theorem I.2. Let $N \subset \pi_{1}^{\text {orb }}(X)$ be the nontrivial normal, cyclic subgroup generated by the fiber of a Seifert fibration of $X$. Unless $X$ is isomorphic to $T^{2} \times I$ or $\bar{T}^{2} \times I, N$ is characteristic.

The next proposition describes the relationship between the orbifold fundamental groups of a Seifert fibered orbifold and its base orbifold.

Proposition I.4 [2, Lemma 4.3]. Suppose that $\pi: X \rightarrow B$ is the projection of a Seifert fibration on an orbifold. Then $\pi$ induces a surjection $\pi_{1}^{\text {orb }}(X) \rightarrow \pi_{1}^{\text {orb }}(B)$. The kernel of this homomorphism is a cyclic normal subgroup generated by the class of a generic fiber in the Seifert fibration. This kernel is nontrivial if $\partial X \neq \varnothing$.

Let $X$ be an orientable 3-orbifold. An edge $e$ in ex $(X)$ is called dihedral if it is of the form:

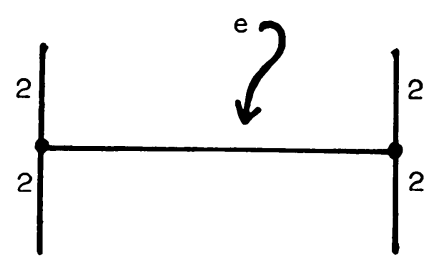


A component of $\operatorname{ex}(X)$ without vertices is called a knot in $\operatorname{ex}(X)$.

If $K$ is a knot in $\operatorname{ex}(X)$ then $\nu K$ will denote a solid torus neighborhood of $K$.

If $K$ is a dihedral edge in $\operatorname{ex}(X)$, then $\nu K$ will denote a neighborhood of $K$ which is isomorphic to
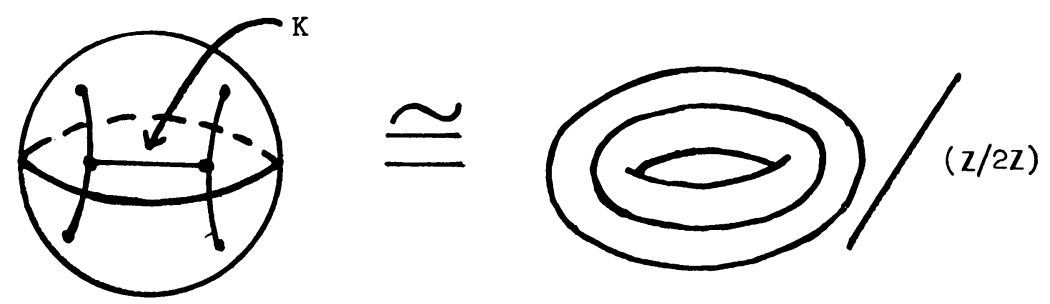

where $\mathbf{Z} / 2 \mathbf{Z}$ acts by a $180^{\circ}$ rotation in an axis piercing the solid torus in two arcs:

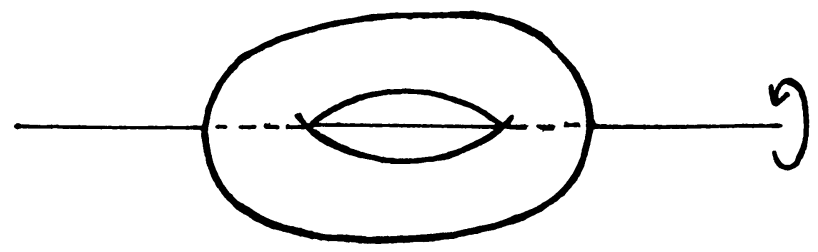

The image in $\nu K$ of a meridian on the solid torus is called a meridian in $\nu K$.

Proposition I.5. Assume that $X$ is an orientable 3-orbifold with finite orbifold fundamental group. Let $K=\amalg K_{i} \subset \operatorname{ex}(X)$ be a set of knots and dihedral edges. If $X-\amalg \nu K_{i}$ is Seifert fibered, then $X$ is Seifert fibered with each $K_{i}$ a fiber.

Proof (analogous to [2, Proposition 7.11]). Let $X-\amalg \nu K_{i} \rightarrow B$ be the fiber map for the fibration on $X-\amalg \nu K_{i}$. It is easy to extend the fibration across $\nu K_{i}$ with $K_{i}$ a fiber if the class of meridian in $\nu K_{i}$ does not project to 1 in $\pi_{1}^{\text {orb }}(B)$. Assume we have done this. Now each meridian projects to 1 . In this case, $\pi_{1}^{\text {orb }}(X)$ surjects onto $\pi_{1}^{\text {orb }}(B)$ and so $B$ is a 2-orbifold with finite orbifold fundamental group. The boundary components of $B$ are of the type:

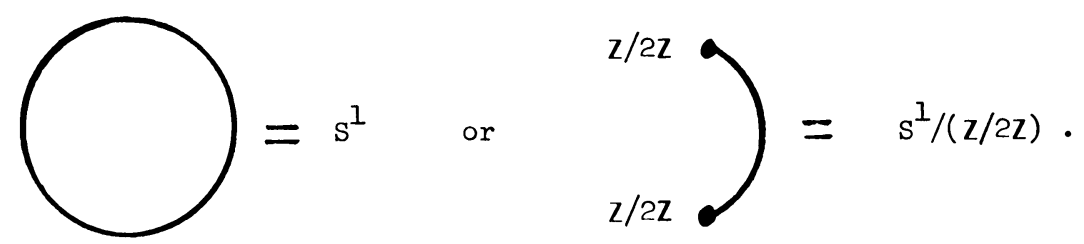

The only possibilities for $B$ up to isomorphism are

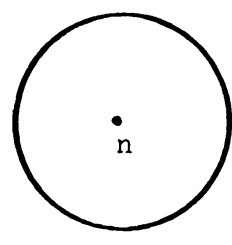

or

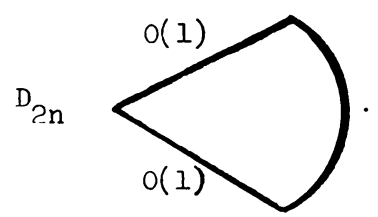


This implies that $X$ is isomorphic to a union of two solid tori with cores as possible exceptional set or that $X$ is isomorphic to a union of two orbifolds of the form $\left(S^{1} \times D^{2}\right) /(\mathbf{Z} / 2 \mathbf{Z})$ where $\mathbf{Z} / 2 \mathbf{Z}$ acts as usual and with the images of the cores as possible additional exceptional set. Both these orbifolds can be Seifert fibered with $K_{i}$ a fiber.

A 2-orbifold is spherical if its universal cover is diffeomorphic to $S^{2}$.

A suborbifold $Y^{d}$ of $X^{n}$ is a subspace $Y \subset X$ locally modelled on quotients of $G \times\left(\mathbf{R}^{n}, \mathbf{R}^{d}\right) \rightarrow\left(\mathbf{R}^{n}, \mathbf{R}^{d}\right)$ where $G$ is a finite subgroup of $O(d) \subset O(n)$.

An orientable 3-orbifold $X$ is prime if every separating spherical suborbifold bounds an orbifold in $X$ which is isomorphic to $D^{3} / G$ for some $G \subset \operatorname{SO}(3)$ acting linearly. Similarly there is the notion of a connected sum of orbifolds.

We circumvent the Poincare conjecture by

Proposition I.6. Assume that $X$ is a closed 3-orbifold of dihedral type with finite orbifold fundamental group. Then $X$ decomposes as $X_{1} \# \Sigma$ where $X_{1}$ is a prime orbifold and $\Sigma$ is a simply connected manifold.

Proof (analogous to [2, Proposition 3.3]). If $X$ is a 3-manifold, this proposition follows from the prime decomposition of 3-manifolds and Van Kampen's theorem, so assume $\operatorname{ex}(X) \neq \varnothing$. If we apply this to $X-\operatorname{ex}(X)$, we obtain $X=X_{1} \# \Sigma$ where $\Sigma$ is a simple connected manifold, $\operatorname{ex}(X)=\operatorname{ex}\left(X_{1}\right)$, and $X_{1}-\operatorname{ex}\left(X_{1}\right)$ is prime. (The prime decomposition for $X-\operatorname{ex}(X)$ yields a decomposition for $X$. There can be only one factor with "boundary" in $X-\operatorname{ex}(X)$ or else Van Kampen's theorem applied to the decomposition for $X$ gives $\pi_{1}^{\text {orb }}(X)$ is infinite. Let $X_{1}$ be the factor $X$ corresponding to the term with "boundary". Let $\Sigma$ be the connected sum of the remaining factors. Again by Van Kampen, $\Sigma$ is simply connected.)

Further, $X_{1}$ is prime. For if $Y^{2} \hookrightarrow X_{1}$ is a separating, spherical suborbifold, then the underlying space of $Y$ is a 2-sphere. Therefore, the orbifold $Y$ is a good (because $X_{1}$ is good), spherical orientable 2-orbifold. Hence $\operatorname{ex}(Y)$ consists of 0 points, 2 points with the same label, or 3 points.

We may assume $\operatorname{ex}(Y)=Y \cap \operatorname{ex}\left(X_{1}\right) \neq \varnothing$ because $X_{1}-\operatorname{ex}\left(X_{1}\right)$ is prime. The orbifold $X_{1}$ is dihedral and so every path of edges labelled 2 in ex $\left(X_{1}\right)$ can be completed to a circuit of edges labelled 2 . Hence if $\operatorname{ex}(Y)$ is 3 points, then they are labelled 2, 2 and $n$. The decomposition $X_{1}=X_{1}^{\prime} \cup_{Y} X_{1}^{\prime \prime}$ induces a decomposition

$$
\pi_{1}^{\text {orb }}\left(X_{1}\right)=\pi_{1}^{\text {orb }}\left(X_{1}^{\prime}\right) *_{\pi_{1}^{\text {orb }}(Y)} \pi_{1}^{\text {orb }}\left(X_{1}^{\prime \prime}\right)
$$

$\pi_{1}^{\text {orb }}(Y) \rightarrow \pi_{1}^{\text {orb }}\left(X_{1}\right)$ injects because $X_{1}$ is good. The fact that $\pi_{1}^{\text {orb }}\left(X_{1}\right)$ is finite implies this decomposition is trivial. Suppose $Y \hookrightarrow X_{1}^{\prime}$ induces an isomorphism of orbifold fundamental groups. By the solution to the Smith conjecture for cyclic or dihedral groups acting on a homotopy 3-disk [3], we know that $X_{1}^{\prime}$ is isomorphic to $\left(D^{3} / G\right) \# \Sigma^{\prime}$ for some $G \subset O(3)$ and $\Sigma^{\prime}$ a homotopy 3-sphere. $X_{1}-\operatorname{ex}\left(X_{1}\right)$ is prime, so $\Sigma^{\prime}$ is isomorphic to $S^{3}$. Thus, $X_{1}^{\prime}$ is isomorphic to $D^{3} / G$ and $X_{1}$ is prime.

In certain cases, the fact that a cover of an orbifold is Seifert fibered implies that the orbifold is Seifert fibered. 
Proposition I.7. Assume that $X$ is a dihedral orbifold with finite orbifold fundamental group. Let $p: Y \rightarrow X$ be a normal orbifold cover. Let $K=\amalg K_{i}$ be a nonempty set of dihedral edges or knots in $\operatorname{ex}(X)$. Assume that $p^{-1}(K) \subset \operatorname{ex}(Y)$. If $Y=Y_{1} \# \Sigma$ where $p^{-1}(K)$ are fibers of a Seifert fibration on $Y_{1}$, and $\Sigma$ is a homotopy 3-sphere, then $X=X_{1} \# \Sigma^{\prime}$ where $X_{1}$ is Seifert fibered with each $K_{i}$ a fiber and $\Sigma^{\prime}$ is a homotopy 3-sphere.

Proof. Let $G$ be the covering isomorphism group of $p: Y \rightarrow S$. The orbifold

$$
Y_{1}-p^{-1}\left(\amalg \nu K_{i}\right)
$$

can be Seifert fibered. Hence, by Proposition I.3, $\pi_{1}^{\text {orb }}\left(Y-p^{-1}\left(\amalg \nu K_{i}\right)\right)$ has a nontrivial, characteristic, cyclic subgroup (in which case $\pi_{1}^{\text {orb }}\left(X-\amalg \nu K_{i}\right)$ has a nontrivial, normal cyclic subgroup) or else $Y_{1}-p^{-1}\left(\amalg \nu K_{i}\right) \cong T^{2} \times I$ or $\bar{T}^{2} \times I$.

Assume that $Y_{1}-p^{-1}\left(\amalg \nu K_{i}\right) \cong T^{2} \times I$ or $\bar{T}^{2} \times I$. We now show that in this case $\pi_{1}^{\text {orb }}\left(X-\amalg \nu K_{i}\right)$ also has a nontrivial, normal, cyclic subgroup. Here, $p^{-1}(K)$ has two connected components and so $K$ has one or two components. Let us do the case where $K$ is a single dihedral edge. The other cases are similar and easier. Let $C$ be one of the two boundary components of $Y-p^{-1}(\nu K)$. Let $H \subset G$ be the stabilizer of $C$. Since $G$ acts transitively on these boundary components, the subgroup $H \subset G$ has index 2 and induces $q: Z \rightarrow X$, a two-sheeted cover. The map $C \hookrightarrow Y-p^{-1}(\nu K)$ induces an isomorphism on orbifold fundamental groups. $C$ is connected and invariant under $H$ and so $\pi_{1}^{\text {orb }}\left(Z-q^{-1}(K)\right)=\pi_{1}^{\text {orb }}\left(Y-p^{-1}(\nu K) / H\right) \cong \pi_{1}^{\text {orb }}(C / H)$. By construction, the orbifold $C / H$ is diffeomorphic to the boundary component of $X-\nu K$. Thus $\pi_{1}^{\text {orb }}\left(Z-q^{-1}(\nu K)\right) \cong \pi_{1}^{\text {orb }}\left(\bar{T}^{2}\right)$. It is easy to see that $\pi_{1}^{\text {orb }}\left(\bar{T}^{2}\right)$ is a semidirect product:

$$
1 \rightarrow \mathbf{Z} \times \mathbf{Z} \rightarrow \pi_{1}^{\text {orb }}\left(\bar{T}^{2}\right) \leftrightarrows \mathbf{Z} / 2 \mathbf{Z} \rightarrow 1
$$

The quotient $\mathbf{Z} / 2 \mathbf{Z}$ acts by $\left(\begin{array}{rl}-1 & 0 \\ 0 & 1\end{array}\right)$. Thus $\pi_{1}^{\text {orb }}\left(Z-q^{-1}(\nu K)\right)$ has a nontrivial, cyclic normal subgroup. Decompose $Z=Z_{1} \# \Sigma^{\prime \prime}$ as in Proposition I.5. Since $\pi_{1}^{\text {orb }}\left(Z_{1}-q^{-1}(\nu K)\right) \cong \pi_{1}^{\text {orb }}\left(Z-q^{-1}(\nu K)\right)$, the space $Z_{1}-q^{-1}(\nu K)$ is diffeomorphic to $\bar{T}^{2} \times I$ by Theorem I.2 and Proposition I.3. We now have another covering,

$$
\left.\left(T^{2} \times I\right) \# \Sigma^{\prime \prime} \# \Sigma^{\prime \prime} \stackrel{2}{\rightarrow} Z-q^{-1}(\nu K)\right) \cong\left(\bar{T}^{2} \times I\right) \# \stackrel{2}{\prime \prime} \stackrel{2}{\rightarrow} X-K .
$$

Since $\mathbf{Z} \times \mathbf{Z}$ is characteristic in $\pi_{1}^{\text {orb }}\left(\bar{T}^{2}\right)$, this sequence of covers gives

$$
1 \rightarrow \mathbf{Z} \times \mathbf{Z} \rightarrow \pi_{1}(X-\nu K) \rightarrow G_{4} \rightarrow 1
$$

where $G_{4}$ is a group of order 4 and we have a homomorphism $G_{4} \stackrel{\varphi}{\rightarrow} \operatorname{Aut}(\mathbf{Z} \times \mathbf{Z})=$ $\mathrm{Gl}_{2}(\mathbf{Z})$. We know that $\left(\begin{array}{cc}-1 & 0 \\ 0 & -1\end{array}\right) \in \operatorname{Im} \varphi$. We are done if we show this action of $G_{4}$ on $\mathbf{Z} \times \mathbf{Z}$ has an invariant, cyclic subgroup. This is true if $\operatorname{Im} \varphi \cong \mathbf{Z} / 2 \mathbf{Z}$ or $\mathbf{Z} / 2 \mathbf{Z} \times$ $\mathbf{Z} / 2 \mathbf{Z}$ because $\left(\begin{array}{cc}-1 & 0 \\ 0 & -1\end{array}\right)$ leaves every cyclic subgroup of $\mathbf{Z} \times \mathbf{Z}$ invariant and every element of order 2 in $\mathrm{Gl}_{2}(\mathbf{Z})$ has an eigenvector. We claim that $\operatorname{Im} \varphi$ is not $\mathbf{Z} / 4 \mathbf{Z}$. For if $G_{4} \cong \mathbf{Z} / 4 \mathbf{Z}$, then the generator is an orientation preserving map which interchanges the boundary tori of $\left(T^{2} \times I\right) \# \Sigma^{\prime \prime} \# \Sigma^{\prime \prime}$ and so acts on $\mathbf{Z} \times \mathbf{Z}$ with determinant -1 . There are no elements of order 4 in $\mathrm{Gl}_{2}(\mathbf{Z})$ with determinant -1 . 
Thus, in all cases we have shown that $\pi_{1}^{\text {orb }}(X-\nu K)$ has a nontrivial, normal, cyclic subgroup. By Theorem I.2 and Proposition I.5, $X=X_{1} \# \Sigma^{\prime}$ where $X_{1}$ is Seifert fibered with each $K_{i}$ a fiber and $\Sigma^{\prime}$ a homotopy 3-sphere.

Let $X$ be a 3 -orbifold. If $X=X_{1} \# \Sigma$ where $X_{1}$ is Seifert fibered and $\Sigma$ is a homotopy 3-sphere, then $X$ is said to be $\sigma$-Seifert fibered.

Corollary I.8. Let $X$ and $K$ be as in Proposition I.7. Let $Y$ be the cover of $X$ corresponding to the fundamental group of its underlying space. If $Y$ is $\sigma$-Seifert fibered, then so is $X$.

Corollary I.9. Let $X$ and $K$ be as in Proposition I.7. Let $Y$ be a cyclic branched cover over a disjoint union of cycles in $\operatorname{ex}(X)$ such that the intersection of $K$ with these cycles consists of vertices. If $Y$ is $\sigma$-Seifert fibered, then so is $X$.

II. The main theorem. The action of a finite group of diffeomorphisms on a homotopy 3-sphere is said to be essentially linear if the quotient orbifold is diffeomorphic to $\left(S^{3} / G\right) \# \Sigma$ where $G \subset O(4)$ acts linearly on $S^{3}$ and $\Sigma$ is a homotopy 3-sphere.

Davis and Morgan prove that the action of a finite group of orientation preserving diffeomorphisms on a homotopy 3-sphere is essentially linear under the hypotheses that all the isotropy subgroups are cyclic and at least one of the isotropy subgroups has order larger than 5. In this section, we extend this result in two directions. We show that such an action is essentially linear if all of the isotropy subgroups are cyclic and one of these subgroups has order 4 . We also show that the action is essentially linear if all of the isotropy subgroups are dihedral or cyclic a long as there is a cyclic isotropy group of order not equal to $1,2,3$ or 5 .

THEOREM II.1. Let $G$ be a finite group of orientation preserving diffeomorphisms of a homotopy 3-sphere $\Sigma$. If all of the isotropy subgroups of $G$ are cyclic or dihedral and there is a cyclic isotropy subgroup of order not 1,2,3 or 5, then the action $G \times \Sigma \rightarrow \Sigma$ is essentially linear.

Davis and Morgan [2] show that the following theorem is equivalent to II.1 (see Theorem I.1).

THEOREM II.2. Assume that $X$ is a prime, closed orbifold of dihedral type with finite orbifold fundamental group. Suppose that there is a point in $X$ whose local group is cyclic of order not 1, 2,3 or 5. Then $X$ is a Seifert fibered orbifold.

First consider a special case.

Lemma II.3. Assume that $X$ is a prime orbifold with finite orbifold fundamental group. Let $\operatorname{ex}(X)$ be a knot $K$ with label 4 . If the underlying space for $X$ is simply connected, then $X$ is Seifert fibered.

Proof. Let $Q$ be the underlying space of $X$. As in the proof of the Smith conjecture [2, section 7], we may assume that $Q-K$ has a hyperbolic structure given by a discrete representation $\pi_{1}(Q-K)=\Gamma \hookrightarrow \operatorname{PSl}_{2}(A) \subset \operatorname{PSl}_{2}(\mathbf{C})$ where $A$ is a ring of algebraic integers in a number field. 
Let $\mu \in \Gamma$ be the class of a meridian about $K$. The element $\mu$ is a parabolic element in $\operatorname{PSl}_{2}(A)$ since it belongs to a subgroup of $\Gamma$ isomorphic to $\mathbf{Z} \times \mathbf{Z}$ and $\Gamma$ is discrete. Let $H=\operatorname{image}\left(\Gamma \rightarrow \operatorname{PSl}_{2}(A / \mathcal{P})\right)$ where $\mathscr{P}$ is a prime ideal in $A$ such that (2) $=\mathscr{P} \cap \mathbf{Z}$. Let $\bar{\mu}$ be the image of $\mu$ in $H$. Since $\mu$ is parabolic, $\bar{\mu}$ has order 2 or 1 . Thus the map $\Gamma \rightarrow H$ factors through a map $\Gamma /\left\{\mu^{4}\right\} \rightarrow H$. The proof of the special case of [2, Theorem 6.3] shows that $H$ cannot be cyclic. The group $H$ is normally generated by $\bar{\mu}$ and so $\bar{\mu}$ has order 2 .

Let $X_{H}$ be the orbifold cover of $X$ corresponding to $H$. The fact that $\bar{\mu}$ has order 2 implies that $\operatorname{ex}\left(X_{H}\right)$ is the preimage of $K$ and has each component labelled 2. So far the proof is the same as in [2].

Consider the action of the cyclic group $\langle\bar{\mu}\rangle \cong \mathbf{Z} / 2 \mathbf{Z}$ on $X_{H}$. Denote the quotient by $\bar{X}_{H}$. The action of the cyclic group generated by the image of $\mu$ in $\pi_{1}^{\text {orb }}(X)$ on the universal cover of $X$ has a point with isotropy subgroup $\mathbf{Z} / 4 Z$. Thus, $\operatorname{ex}\left(\bar{X}_{H}\right)$ has a component labelled 4. By taking covers corresponding to the fundamental group of underlying spaces and cyclic branched covers, we obtain a cover $Z$ of $\bar{X}_{H}$ whose underlying space is simply connected and whose exceptional set consists of a knot labelled 4. The orbifold fundamental group of $Z$ has order smaller than the order of $\pi_{1}^{\text {orb }}(X)$ because $H$ is not cyclic.

If $Z$ were not $\sigma$-Seifert fibered, we could repeat the above construction. The order of $\pi_{1}^{\text {orb }}(X)$ is finite and so one of these covers will be $\sigma$-Seifert fibered. Therefore, by induction on the order of the orbifold fundamental group we may assume that $Z$ is $\sigma$-Seifert fibered. By Corollaries I.8 and I.9, $\bar{X}_{H}=\bar{X}_{H}^{\prime} \# \Sigma$ is $\sigma$-Seifert fibered. We now arrange the fibration on $\bar{X}_{H}^{\prime}$ so that $\operatorname{ex}\left(\bar{X}_{H}\right)$ consists of fibers. If this is not the case, then the base orbifold $B$ for the fibration has a point with local group $O(1)$. This base also has a point with local group cyclic of order $n \geqslant 4$ because $\operatorname{ex}\left(\bar{X}_{H}\right)$ has a component labelled 4 . The map $\pi_{1}^{\text {orb }}\left(\bar{X}_{H}^{\prime}\right) \rightarrow \pi_{1}^{\text {orb }}(B)$ is a surjection and so $\pi_{1}^{\text {orb }}(B)$ is finite. The only possibility for $B$, up to isomorphism, is

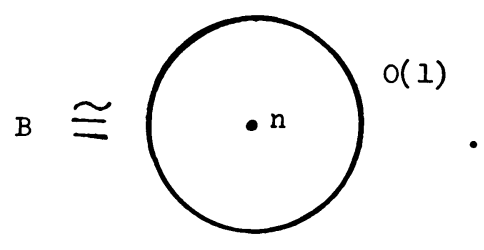

Decompose $B$ as indicated by

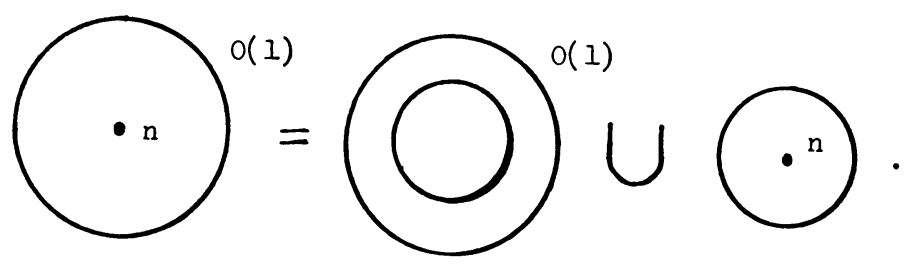

We see that $\bar{X}_{H}^{\prime}$ is the union of two solid tori. The exceptional set of $\bar{X}_{H}^{\prime}$ consists of the boundary of a twisted band running along the core of one of the tori (this boundary is labelled 2) together with the core of the other torus labelled 4 . In this exceptional case, it is easy (using Proposition I.5) to refiber $\bar{X}_{H}^{\prime}$ so that $\operatorname{ex}\left(\bar{X}_{H}\right)$ consists of fibers. 
Because Seifert fibrations lift to Seifert fibrations, we can realize a $\sigma$-Seifert fibration on $X_{H}=X_{H}^{\prime} \# \Sigma^{\prime}$ with ex $\left(X_{H}\right)$ consisting of fibers. By Proposition I.7, $X$ is $\sigma$-Seifert fibered but $X$ is prime and so $X$ is Seifert fibered.

Now we prove Theorem II.2. By taking covers corresponding to the fundamental group of the underlying space or cyclic-branched covers of the type described in Corollary I.9, we may obtain from $X$ a cover with simply connected underlying space and exceptional set consisting of a single knot with label not equal to 1,2,3 or 5 . This cover is $\sigma$-Seifert fibered by Lemma II.3 and the main result of Davis and Morgan [2]. Proposition I.7 then implies that $X$ is $\sigma$-Seifert fibered. $X$ is prime and so $X$ is Seifert fibered.

The proof given here of Lemma II.3 works as well in the case where $K$ has label any composite number. It does not depend on a knowledge of the subgroups of $\mathrm{PSl}_{2}(A / \mathfrak{p})$.

For a sketch of the proof of the Smith conjecture and the results in Davis and Morgan [2], see Morgan [4].

\section{REFERENCES}

1. M. Culler and P. B. Shalen, Varieties of group representations and splittings of 3-manifolds, Ann. of Math. (2) 117 (1983), 109-146.

2. M. W. Davis and J. W. Morgan, Finite group actions on homotopy 3-spheres, the volume on the Smith conjecture (to appear).

3. W. Meeks, III and S. T. Yau, Group actions on $\mathbf{R}^{3}$, the volume on the Smith conjecture (to appear).

4. J. W. Morgan, Actions de groupes finis sur $S^{3}$ : la conjecture de P. A. Smith (d'apres Thurston et Meeks-Yau), Sémin. Bourbaki 578, Lecture Notes in Math., vol. 901, Springer-Verlag. Berlin and New York, pp. 277-289.

5. W. Thurston, The geometry and topology of 3-manifolds (preprint).

Department of Mathematics, University of California, Los Angeles, California 90024 